Draft Version August 15, 2019

Preprint typeset using LTEX style emulateapj v. 12/16/11

\title{
V348 AND, AND V572 PER: BRIGHT TRIPLE SYSTEMS WITH ECCENTRIC ECLIPSING BINARIES
}

\author{
P. Zasche ${ }^{1}$, R. Uhla $\check{R}^{2}$, P. Svoboda ${ }^{3}$, J. JuryšeK ${ }^{4}$, D. KorčÁKová ${ }^{1}$, M. Wolf ${ }^{1}$, M. Šlechta $^{5}$, L. Kotková ${ }^{5}$ \\ ${ }^{1}$ Astronomical Institute, Charles University, Faculty of Mathematics and Physics, CZ-180 00, Praha 8, V Holešovičkách 2, Czech Republic, \\ ${ }^{2}$ Private Observatory, Pohoří 71, CZ-254 01 Jílové u Prahy, Czech Republic \\ ${ }^{3}$ Private Observatory, Výpustky 5, CZ-614 00, Brno, Czech Republic \\ ${ }^{4}$ Institute of Physics, The Czech Academy of Sciences, Na Slovance 1999/2, CZ-182 21, Praha, Czech Republic \\ ${ }^{5}$ Astronomical Institute, The Czech Academy of Sciences, CZ-251 65, Ondřejov, Czech Republic
}

(Dated: Received 2019 April 2; revised 2019 June 3; accepted 2019 June 25; published 2019 August 2) Draft version August 15, 2019

\begin{abstract}
The eclipsing binaries are still important objects for our understanding of the Universe. Especially these ones located within the more complex multiple systems can help us solving the problem of their origin and subsequent evolution of these higher-order multiples. Photometry and spectroscopy spanning over more than 25 years were used for the first complete analysis of the two bright triple systems, namely V348 And and V572 Per. The light curves in photometric filters were combined together with the radial velocities and analysed simultaneously, yielding the precise physical parameters of the eclipsing components of these multiple systems. The system V348 And consists of two eclipsing components with its orbital period of about 27.7 days. The system is a very detached one, and both eclipses are rather narrow, lasting only about 0.016 of its period. The visual orbit of the wide pair has the period of about 87 years. All three components of the system are probably of B8-9 spectral type, and the parallax of the system was slightly shifted to the value of 2.92 mas. On the other hand, the system V572 Per shows apsidal motion of its inner orbit, the orbital period being of about 1.2 days, while the apsidal motion of about 48 years. The components are of $\mathrm{A}$ and $\mathrm{F}$ spectral types, while the motion with the third component around a common barycenter is only negligible. According to our modelling, this system is not a member of open cluster Alpha Persei.

Subject headings: stars: binaries: eclipsing - stars: binaries: visual - stars: binaries: spectroscopic - stars: fundamental parameters - stars: individual: V348 And, V572 Per - open clusters and associations: individual: Alpha Persei Cluster
\end{abstract}

\section{INTRODUCTION}

The eclipsing binaries still represent the most general method how to derive the stellar masses, radii and luminosities most precisely. The fruitful combination of the observational techniques like photometry and spectroscopy is still being used also for deriving the temperatures, surface gravities or limb darkening, but also to compute the distances to these systems.

On the other hand, studying the binaries as parts of the higher order multiple systems can bring us new important results connected with the stellar origin and evolution. We can ask - how many multiple systems are there within the stellar population? What is the multiplicity fraction of the field stars, and is this number still the same? Or is it somehow evolving in time and the multiplicity fraction can be tracked as different between PopI and PopIII stars? Is the mass ratio, period ratio, or eccentricity ratio the same for the low mass as well as for the high mass stars? What is the role of the Kozai cycles (see e.g. Eggleton \& Kiseleva-Eggleton 2001)? Can the effects like synchronization or circularization predicted by the tidal theories (Zahn 2008) be traced in particular systems via deriving their orbital and physical parameters? These and many other still open questions play a crucial role in our theories of stellar formation and evolution, see e.g. Tokovinin (2008), Halbwachs et al. (2003), or Tokovinin (2014). And of course, the models can be tested and verified only when using the real data, which can be obtained only via studying the par-

zasche@sirrah.troja.mff.cuni.cz ticular system and derive its parameters. The importance of dedicated studies of particular systems with higher hierarchies was presented e.g. in the updated version of the Multiple Star Catalog MSC, Tokovinin (2018). This is the main aim of the present paper, to bring new results on two new multiple systems never studied before. And moreover, both these systems are bright enough for subsequent follow-up observations.

Due to this reason, we have focused our effort on two systems for which their light curves (hereafter LC) and radial velocity curves were not studied yet (namely V348 And, and V572 Per). Besides the inner eclipsing pair, both these systems also contain the distant third component detected via interferometry with its rather long orbital period. Moreover, both these stars show the eccentric orbits.

\section{THE ANALYSIS}

Whole our analysis is using a classical combination of the photometry and spectroscopy into one joint approach. If we combine these methods, we can obtain the physical parameters of both eclipsing components as well as the parameters of their mutual orbit. As a consequence, having the complete information about their masses, inclinations, periods, etc. we can also fill in still quite incomplete statistics of the multiple (triple and quadruple) systems. All of these distributions of orbital and physical parameters are being used for discussions about the origin and subsequent evolution of such multiples (Tokovinin 2008 and Tokovinin 2014).

All of our new spectroscopic observations were secured in the Ondřejov observatory in Czech Republic, using the 2meter telescope. The classical slit spectrograph has its res- 
olution $\mathrm{R} \sim 12500$. The individual exposure times were chosen according to the quality of the particular night, typically 800-3600 seconds. All the spectrograms were reduced in a standard way, the wavelength calibration was made via a ThAr comparison spectra obtained before and after the stellar ones. The flatfields were taken in the beginning and end of the night and their averages were then used for the reduction. After then, the radial velocities (hereafter RV) were derived with the program SPEFO (Horn et al. 1996, or Śkoda 1996), on several absorption lines in the measured spectral region around $\mathrm{H}_{\alpha}$ (usually $\mathrm{Fe}, \mathrm{Ca}$, or $\mathrm{Si}$ lines), with using the zero point correction via measuring the telluric lines.

Owing to relatively high brightness of these stars, only rather small telescopes were used for the photometric observations. The system V348 And was observed (by PS) with only the 34-mm refractor at his private observatory in Brno, Czech Republic, using the SBIG ST-7 CCD camera. The second star V572 Per was monitored with the similar instrument at the private observatory (by RU) in Jílové u Prahy, Czech Republic, using a G2-0402 CCD camera. All the measurements were reduced in a standard way using the programme C-MUNIPACK $\square$ which is based on aperture photometry and uses the standard DAOPHOT procedures (Tody 1993). The photometric data were obtained during the time span $2007-$ 2018. Nevertheless, some of the older data were only used for the minima times derivation. All of these data were secured in the Johnson-Cousins photometric system (Bessell 1990), particularly the system V348 And was observed in $B V R$, while the system V572 Per in BVRI filters.

Both photometric and spectroscopic observations were studied in the standard approach. Hence, the program PHOEBE (Prša \& Zwitter 2005), which is using the classical Wilson-Devinney algorithm (Wilson \& Devinney (1971) and its later modifications) was used for the analysis. It allows us to fit the relevant physical parameters of the eclipsing components, as well as their relative orbit. For the analysis, we used several assumptions. At first, the primary temperature was set to the value corresponding to the particular spectral type (see calibrations by Pecaut \& Mamajek (2013) and the more updated web site $)$. The coefficients of the limb-darkening were interpolated from van Hamme's tables (van Hamme 1993). The coefficients of albedo $A_{i}$, and the gravity darkening coefficients $g_{i}$ were fixed at their suggested values. And finally, the third light was also computed because we deal with the triple systems and the additional light can be easily attributed to this distant component.

The errors of individual parameters were derived via combined approach using besides the PHOEBE also the code called Pyterpor 3 (Nemravová et al. 2016). It derives the radiative and kinematic parameters of components via comparison of observed spectra to the synthetic ones.

For the fitting of apsidal motion and visual orbit (see below) our own codes were used. These programs are using the weighted least squares method and the simplex algorithm. For error estimation the boot-strapping method was used.

\section{V348 AND}

The first system is the northern-hemisphere star V348 And (= HIP 1233, HD 1082, $\left.V_{\max }=6.6 \mathrm{mag}\right)$. Since Dyson (1935) its spectral type is usually being classified as B9. Despite

\footnotetext{
${ }^{1}$ See http://c-munipack.sourceforge.net/

${ }^{2}$ www.pas.rochester.edu/ emamajek/EEM_dwarf_UBVIJHK_colors_Teff.txt

${ }^{3}$ https://github.com/chrysante87/pyterpol/wiki
}

its brightness and northern declination, its detailed analysis is still missing. The star was classified as an eclipsing binary based on the Hipparcos data (Perryman \& ESA 1997), having the supposed orbital period of about 5.5 days. However, according to our observations obtained during 2007-2008 we found there no evidence of eclipse. Hence, we prematurely stated that the star was incorrectly classified as an eclipsing binary, see our previous paper on this star in Zasche \& Svoboda (2008).

According to our new findings presented in this paper, we found that the star is eclipsing, but with much longer orbital period. Moreover, the star is also known as a visual binary, having the distant component of about $0.1^{\prime \prime}$ away from the primary. However, its orbital period is still rather uncertain (see below). The parallax of the system was derived as 3.02 mas (van Leeuwen 2007), while more recently by GAIA Gaia Collaboration et al. 2018) as 2.16 mas.

After several years of observations, there was detected a photometric decrease, indicating that the star is really an eclipsing one. And finally after many other nights of observations we found out that its period is much longer than previously assumed, being of about 27.7 days. However, these eclipses are very narrow (lasting about only 0.016 of the phase, which is of about 10.4 hours), but relatively deep, of about 0.14 mag in $R$ filter.

The combined analysis of LC+RV provides us an insight into basic physical parameters of both components. System is composed of two very similar stars. Primary and secondary components are both of B9V spectral type. The results of our analysis are given in Table 1, while the plots of the RV curve as well as the light curves are given in Fig. 11 The third component is even more luminous than the eclipsing pair itself, and according to the spectra its spectral type should be similar, of about B8V. As one can see, the eccentricity of the orbit is only small, while the $\omega$ angle remains practically the same, hence the apsidal motion is only very slow (longer than $1000 \mathrm{yr}$ ). For this eclipse-times analysis of apsidal motion we collected all available times of eclipses, our new observed ones as well as those derived from photometry by Hipparcos (Perryman \& ESA 1997), INTEGRAL/OMC (Mas-Hesse et al. 2003), and MASCARA (Burggraaff et al. 2018). All of these are given below in Table 3 .

Thanks to its brightness and period, both the masses and radii can be derived very precisely at the level of $2 \%$ only. Hence, we can state that there is no other similar longperiod $(\mathrm{P}>20 \mathrm{~d})$ system with main-sequence components in our Galaxy with such well-derived parameters (see the DEBCat catalogue by Southworth 2015).

Besides the photometry and spectroscopy, also the visual orbit of V348 And (i.e. WDS J00153+4412AB) was recomputed. Our new solution (see Table 2) differs from the already published solutions. Both Seymour et al. (2002) and Olevic et al. (2003) presented much longer orbits. But according to our modelling, there is a need for tighter orbit due to its total mass. As one can compute from both eclipsing components, and using the Hipparcos parallax, the total mass of the system is of about $8.1 \mathrm{M}_{\odot}$. Subtracting the masses of primary and secondary, the tertiary mass should have of about $2.6 \mathrm{M}_{\odot}$. But such a small mass is unrealistic for such a luminous body. Hence, our final conclusion is that the total mass of the system is of about $8.9 \mathrm{M}_{\odot}$ (i.e. $2.8+2.7+3.4$ ), but the system is located slightly more away, i.e. having the parallax of 2.92 mas. Such a value is still within the error interval of the Hipparcos value, but outside of the new GAIA parallax. 

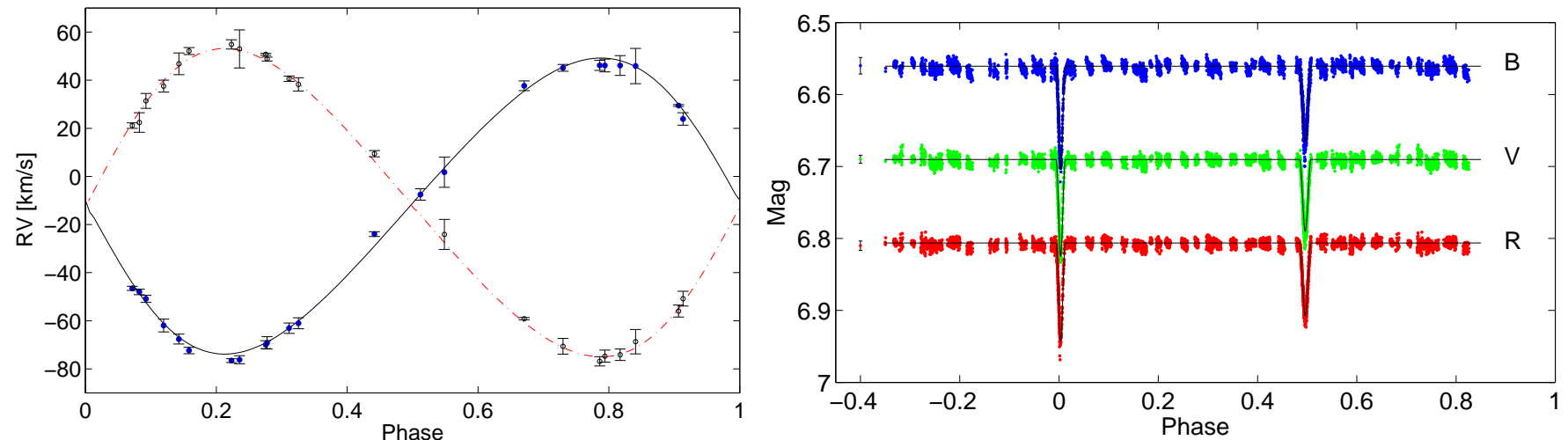

FIG. 1.- Results of our LC+RV analysis of V348 And as based on the PHOEBE fitting. Left: For the radial velocities, the primary is plotted as a solid line (and full dots), while secondary as a dashed line (and open circles). Right: For the photometry the data collected during more than 10 years. Individual curves were shifted in $y$-axis for better brevity of the picture. Typical precision of individual observations are also plotted as short error bars on the left.

TABLE 1

THE PARAMETERS FROM THE LC+RV FITTING OF BOTH SYSTEMS.

\begin{tabular}{c|ccc|ccc}
\hline \hline & \multicolumn{3}{|c|}{ V348 And } & \multicolumn{3}{c}{ V572 Per } \\
Parameter & Primary & Secondary & Tertiary & Primary & Secondary & Tertiary \\
\hline$H J D_{0}$ & $2455923.129 \pm 0.025$ & - & $2457007.3701 \pm 0.0017$ & - \\
$P[\mathrm{~d}]$ & $27.703514 \pm 0.000325$ & - & $1.2131789 \pm 0.0000006$ & - \\
$a\left[\mathrm{R}_{\odot}\right]$ & $68.28 \pm 0.87$ & - & $7.01 \pm 0.07$ & - \\
$v_{\gamma}[\mathrm{km} / \mathrm{s}]$ & $-11.63 \pm 0.52$ & - & $-0.05 \pm 0.20$ & - \\
$e$ & $0.116 \pm 0.012$ & - & $0.029 \pm 0.002$ & - \\
$\omega[\mathrm{deg}]$ & $95.6 \pm 0.2$ & - & $10.0 \pm 0.1$ & - \\
$\omega[\mathrm{deg} / \mathrm{yr}]$ & \multicolumn{2}{|c|}{-} & - & $7.5 \pm 0.9$ & - \\
$q=M_{2} / M_{1}$ & $0.96 \pm 0.02$ & - & $0.79 \pm 0.02$ & - \\
$i[\mathrm{deg}]$ & $88.26 \pm 0.02$ & - & $80.7 \pm 0.8$ & - \\
$K[\mathrm{~km} / \mathrm{s}]$ & $61.22 \pm 0.81$ & $63.76 \pm 0.85$ & - & $126.73 \pm 2.0$ & $160.41 \pm 2.3$ & - \\
$T[\mathrm{~K}]$ & $10500($ fixed) & $10412 \pm 87$ & - & $8000(\mathrm{fixed})$ & $6654 \pm 112$ & - \\
$M\left[\mathrm{M}_{\odot}\right]$ & $2.81 \pm 0.04$ & $2.69 \pm 0.04$ & - & $1.76 \pm 0.03$ & $1.39 \pm 0.03$ & - \\
$R\left[\mathrm{R}_{\odot}\right]$ & $2.42 \pm 0.03$ & $2.34 \pm 0.03$ & - & $1.68 \pm 0.03$ & $1.29 \pm 0.04$ & - \\
$M_{b o l}[\mathrm{mag}]$ & $0.23 \pm 0.01$ & $0.33 \pm 0.01$ & - & $2.21 \pm 0.05$ & $3.57 \pm 0.07$ & - \\
$L_{B}[\%]$ & $23.5 \pm 0.6$ & $21.6 \pm 0.9$ & $54.9 \pm 1.3$ & $68.5 \pm 0.9$ & $14.7 \pm 0.5$ & $16.7 \pm 1.1$ \\
$L_{V}[\%]$ & $22.3 \pm 0.7$ & $20.5 \pm 0.8$ & $57.2 \pm 1.7$ & $63.0 \pm 1.0$ & $17.0 \pm 0.7$ & $20.0 \pm 0.8$ \\
$L_{R}[\%]$ & $22.7 \pm 0.6$ & $20.9 \pm 0.7$ & $56.4 \pm 1.0$ & $60.4 \pm 0.8$ & $19.1 \pm 0.5$ & $20.5 \pm 1.0$ \\
$L_{I}[\%]$ & - & - & - & $55.2 \pm 0.7$ & $20.2 \pm 0.6$ & $24.6 \pm 0.9$ \\
\hline
\end{tabular}

TABLE 2

THE PARAMETERS OF VISUAL ORBIT OF V348 AND.

\begin{tabular}{cccc}
\hline \hline Parameter & Our solution & Seymour et al. (2002) & Olevic et al. (2003) \\
\hline$p_{3}[\mathrm{yr}]$ & $86.9 \pm 4.3$ & 330 & $137.958 \pm 1.657$ \\
$T_{0}$ & $2451501 \pm 96$ & 2449900 & $2450734.4 \pm 43.8$ \\
$e$ & $0.559 \pm 0.015$ & 0.715 & $0.7238 \pm 0.0096$ \\
$a$ [arcsec] & $0.118 \pm 0.013$ & 0.29 & $0.1527 \pm 0.0008$ \\
$i$ [deg] & $66.0 \pm 3.9$ & 73.8 & $62.9 \pm 0.3$ \\
$\Omega[\mathrm{deg}]$ & $61.1 \pm 2.4$ & 64.3 & $68.4 \pm 1.0$ \\
$\omega[\mathrm{deg}]$ & $139.7 \pm 10.2$ & 78 & $118.14 \pm 0.02$ \\
\hline
\end{tabular}

We can only speculate that there can be a problem with deriving the parallax value when the instrument is not able to resolve the double into two separate targets and this can shift the true value of parallax.

\section{V572 PER}

The second system in our study is called V572 Per (= HIP 15193, HD 20096, $\left.V_{\max }=6.7 \mathrm{mag}\right)$. It is also a detached binary, but it was never studied before. Its photometric variability was also recognized by the Hipparcos (Perryman \& ESA 1997), having the orbital period of about 1.21 days. Similarly to V348 And it also contains close vi- sual component. However, this component (being of about $\left.1.5^{\prime \prime}\right)$ is not moving noticeably on the sky, hence any reliable third-body orbit cannot be derived. Moreover, the star was also proved as a member of close open cluster Alpha Persei (Zuckerman et al. 2012). The GAIA survey Gaia Collaboration et al. 2018) provided new measurement of its parallax, however it gives two numbers for two close sources. The brighter one $8.07 \pm 0.05$ mas, while the fainter one $7.97 \pm 0.06$ mas (i.e. the distance $123-126 \mathrm{pc}$ ).

Results of our LC+RV fitting are given in Table 1 while the plots of RV and LC are presented in Fig. 3.

Moreover, besides the LC+RV fitting, we also performed 


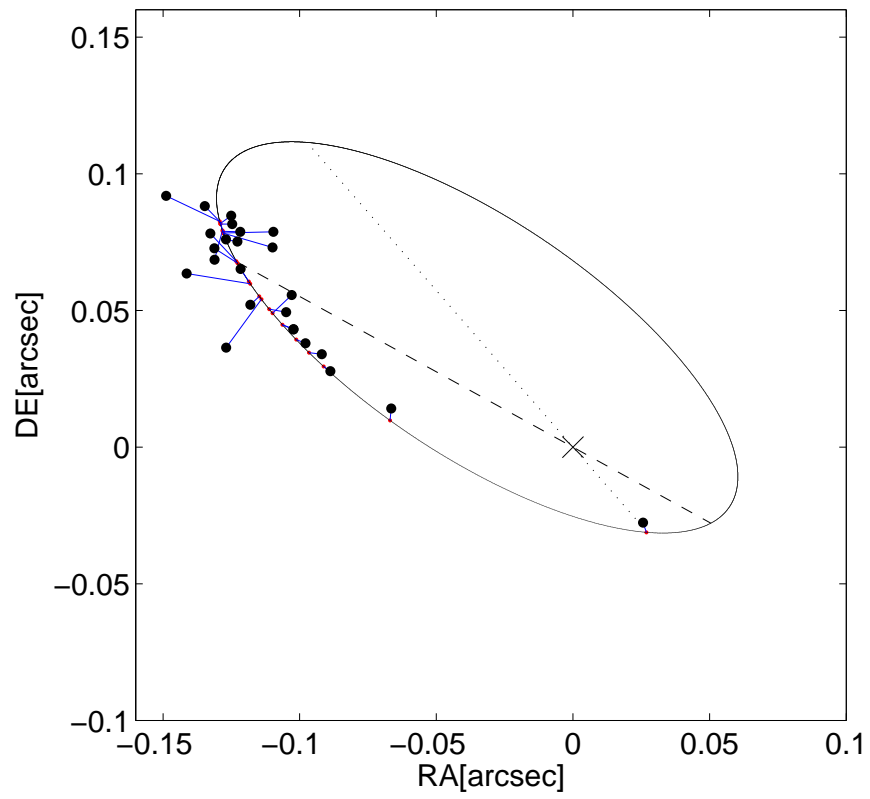

FIG. 2.- Visual orbit of V348 And. The individual observations are connected with their theoretical positions on the orbit, while the dotted line stands for the line of the apsides and the dashed line stands for the line of the nodes.

an analysis of the minima times of V572 Per, which revealed that the system shows an apsidal motion of its eccentric orbit. Such a movement is plotted in Fig. 4 where the apsidal motion during almost 30 years is clearly visible. Its period is of about 48 years only, hence the system deserves special attention and new photometric observations in the upcoming years to confirm this apsidal motion. Relativistic contribution to the total apsidal motion rate is of about $4 \%$. An internal structure constant resulted in $\log k_{2}=-2.37$, which can be compared with the tables e.g. by Claret (2004). There resulted that the system is still rather young now, having the age of about 20 Myr only.

From the combined analysis of $\mathrm{LC}+\mathrm{RV}$ there also resulted that the third component in the light curve contributes of about $20 \%$ to its total luminosity (depending on the photometric filter). This yielded a magnitude difference between the combined light of an eclipsing pair and this third component of about $1.5 \mathrm{mag}$. Such a result is in perfect agreement with the values given in the $4^{\text {th }}$ Catalog of Interferometric Measurements of Binary Stars (Hartkopf et al. 2001), which are in the range from 1.48 to $1.65 \mathrm{mag}$.

We can also compute the photometric distance to the system using our derived values. At this point we found a problem. According to different published papers on open cluster Alpha Persei (e.g. Jackson \& Jeffries 2010, Robichon et al. 1999, or Pinsonneault et al. 1998), its distance modulus is probably in between 6.0 and $6.5 \mathrm{mag}$ (i.e. the distance $158-200 \mathrm{pc}$ ). And according to Makarov (2006) the star is a member of that cluster. However, as one can check from its proper motion and distance, it is quite disputable member of such cluster. According to our result, the distance modulus resulted in about 5.0 mag (i.e. $100 \mathrm{pc}$ distant), which means the star is much closer to the Sun than the cluster itself and is very probably not connected with it. The value of GAIA parallax provides a distance in between these two distances.

\section{DISCUSSION AND CONCLUSIONS}

In the era of huge surveys both on photometry and spectroscopy, one can ask whether a dedicated study on one particular binary is still worth of an effort. However, as was presented quite recently (Kim et al. 2018), the catalogue of eclipsing binaries with eccentric orbits and apsidal motion is still only sparsely populated with stars having the complete LC+RV solution (hence with precisely derived masses) with periods longer than 20 days. Our study of V348 And can serve as a good example. Moreover, its parameters were derived with high precision at a level of about $2 \%$. The other system V572 Per can enrich a still limited group of short eccentric systems with fast apsidal motion ( $\mathrm{U}<50 \mathrm{yr}$ ), which nowadays comprises only 21 systems (Kim et al.2018). Hence any new contribution, moreover with derived masses, is welcome. And as was mentioned in many other publications (like e.g. Claret \& Giménez 2010) their role for testing the stellar structure theories is still undisputable.

Moreover, besides the eccentric inner orbit, also the fact that we deal with hierarchical triple systems is of high importance. As was presented recently e.g. by Tokovinin (2014), there is an excess of tight inner binaries within the triples, probably caused by tidal evolution and Kozai cycles.

It also should be noted that both these stars are of high brightness and also located on the northern sky. One would expect that almost all of the interesting systems here are known and were studied before. But, as we have pointed out, also some interesting results still can be obtained with very small photometric instruments (all photometry for our study was obtained by using only the small telescopes having the aperture of less than $8 \mathrm{~cm}$ ).

We would like to thank Ms. J.A.Nemravová, Mr. J. Čechura, and Mr. R. Kř́čck for their contribution to the spectroscopic observations of both targets. An anonymous referee is also to be acknowledged for his/her valuable comments improving the manuscript. This research has made use of the Washington Double Star Catalog maintained at the U.S. Naval Observatory. This investigation was supported by the Czech Science Foundation grants No. P209/10/0715, GA1502112S, and GA17-00871S. Work is partly based on the data from the OMC Archive at CAB (INTA-CSIC), pre-processed by ISDC. This research has made use of the SIMBAD and VIZIER databases, operated at CDS, Strasbourg, France and of NASA's Astrophysics Data System Bibliographic Services.

\section{REFERENCES}

Bessell, M. S. 1990, PASP, 102, 1181

Burggraaff, O., Talens, G. J. J., Spronck, J., et al. 2018, A\&A, 617, A32 Claret, A. 2004, A\&A, 424, 919

Claret, A. \& Giménez, A. 2010, A\&A, 519, A57

Dyson, F. W. 1935, Second Greenwich Catalogue of stars for 1925.0 from obs. with the transit circle 1922-30. Second part: Catalogue of stars in the zone +32 to +64 degree obs. 1922 - 30; p. 45 .

Eggleton, P. P. \& Kiseleva-Eggleton, L. 2001, ApJ, 562, 1012
Gaia Collaboration, Brown, A. G. A., Vallenari, A., et al. 2018, A\&A, 616, A1

Halbwachs, J. L., Mayor, M., Udry, S., \& Arenou, F. 2003, A\&A, 397, 159 Hartkopf, W. I., McAlister, H. A., \& Mason, B. D. 2001, AJ, 122, 3480 Horn, J., Kubát, J., Harmanec, P., et al. 1996, A\&A, 309, 521

Jackson, R. J. \& Jeffries, R. D. 2010, MNRAS, 402, 1380

Kim, C.-H., Kreiner, J. M., Zakrzewski, B., et al. 2018, ApJS, 235, 41 Makarov, V. V. 2006, AJ, 131, 2967

Mas-Hesse, J. M., Giménez, A., Culhane, J. L., et al. 2003, A\&A, 411, L261 


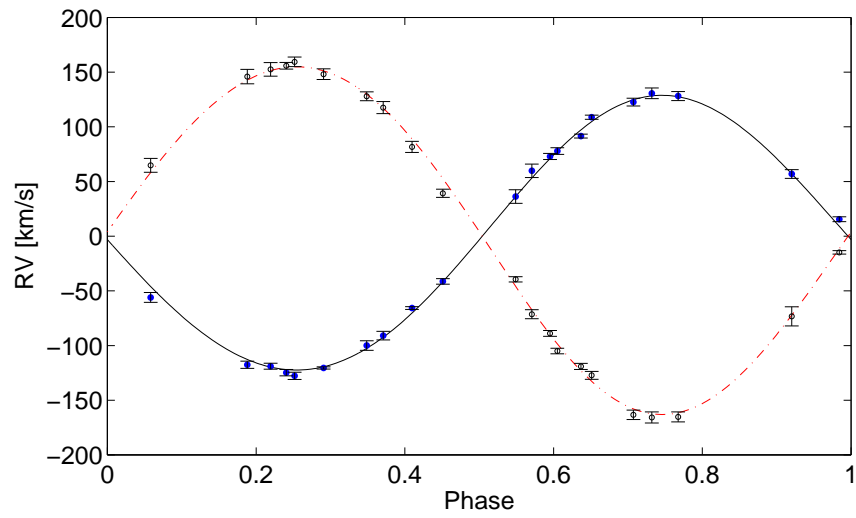

FIG. 3. - Result of our RV+LC fitting of V572 Per, notation same as in Fig. 1

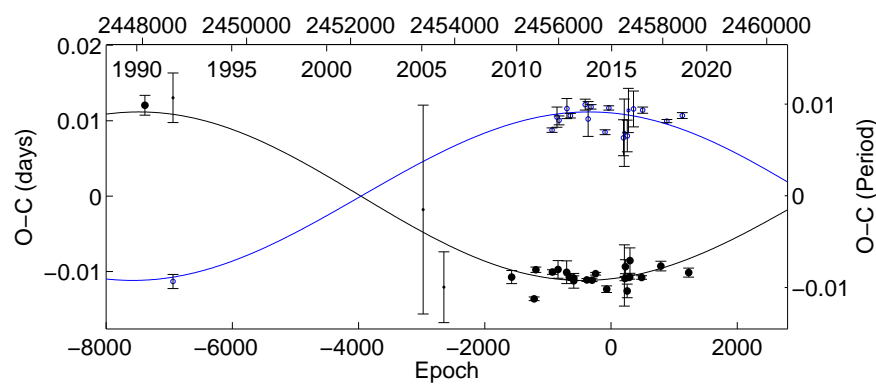

FIG. 4.- Variations of the minima times plotted in the $O-C$ diagram. Black solid line and the full dots stand for primary eclipses, while the blue line and the open circles represent the secondary ones.

Nemravová, J., Harmanec, P., Brož, M., et al. 2016, A\&A, 594, A55 Olevic, D., Popovic, G. M., Pavlovic, R., \& Cvetkovic, Z. 2003, Serbian Astronomical Journal, 166

Pecaut, M. J. \& Mamajek, E. E. 2013, ApJS, 208, 9

Perryman, M. A. C. \& ESA. 1997, The HIPPARCOS and TYCHO

catalogues (The Hipparcos and Tycho catalogues. Astrometric and photometric star catalogues derived from the ESA Hipparcos Space Astrometry Mission, Publisher: Noordwijk, Netherlands: ESA Publications Division, 1997, Series: ESA SP Series 1200)

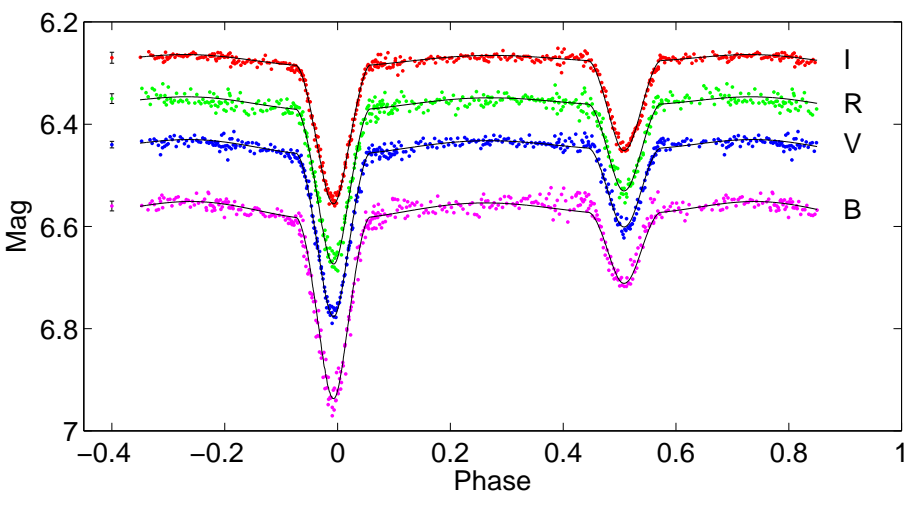

Pinsonneault, M. H., Stauffer, J., Soderblom, D. R., King, J. R., \& Hanson, R. B. 1998, ApJ, 504, 170

Prša, A. \& Zwitter, T. 2005, ApJ, 628, 426

Robichon, N., Arenou, F., Mermilliod, J.-C., \& Turon, C. 1999, A\&A, 345, 471

Seymour, D. M., Mason, B. D., Hartkopf, W. I., \& Wycoff, G. L. 2002, AJ, 123,1023

Škoda, P. 1996, in ASPC, Vol. 101, Astronomical Data Analysis Software and Systems V, ed. G. H. Jacoby \& J. Barnes, 187

Southworth, J. 2015, in Astronomical Society of the Pacific Conference Series, Vol. 496, Living Together: Planets, Host Stars and Binaries, ed. S. M. Rucinski, G. Torres, \& M. Zejda, 164

Tody, D. 1993, in ASPC, Vol. 52, Astronomical Data Analysis Software and Systems II, ed. R. J. Hanisch, R. J. V. Brissenden, \& J. Barnes, 173

Tokovinin, A. 2008, MNRAS, 389, 925

Tokovinin, A. 2014, AJ, 147, 87

Tokovinin, A. 2018, ApJS, 235, 6

van Hamme, W. 1993, AJ, 106, 2096

van Leeuwen, F. 2007, A\&A, 474, 653

Wilson, R. E. \& Devinney, E. J. 1971, ApJ, 166, 605

Zahn, J.-P. 2008, in EAS Publications Series, Vol. 29, EAS Publications Series, ed. M.-J. Goupil \& J.-P. Zahn, 67-90

Zasche, P. \& Svoboda, P. 2008, Information Bulletin on Variable Stars, 5827,1

Zuckerman, B., Melis, C., Rhee, J. H., Schneider, A., \& Song, I. 2012, ApJ, 752,58

\section{ONLINE-ONLY MATERIAL}


TABLE 3

HELIOCENTRIC MINIMA OF THE SYSTEMS USED FOR OUR ANALYSIS.

\begin{tabular}{|c|c|c|c|c|c|}
\hline $\begin{array}{l}\text { Star } \\
\text { name }\end{array}$ & $\begin{array}{l}\text { HJD - } 2400000 \\
\text { [d] }\end{array}$ & $\begin{array}{l}\text { Error } \\
\text { [d] }\end{array}$ & Type & Filter & Reference \\
\hline V348 And & 48498.65159 & 0.00627 & $\bar{P}$ & $\mathrm{Hp}$ & Hipparcos \\
\hline V348 And & 54482.66675 & 0.04519 & $P$ & $\mathrm{~V}$ & OMC/INTEGRAL \\
\hline V348 And & 57142.16495 & 0.00016 & $\mathrm{P}$ & $\mathrm{C}$ & MASCARA \\
\hline V348 And & 57155.82545 & 0.00834 & S & $\mathrm{C}$ & MASCARA \\
\hline V348 And & 57252.99676 & 0.00107 & $P$ & $\mathrm{C}$ & MASCARA \\
\hline V348 And & 57266.64006 & 0.00222 & S & $\mathrm{C}$ & MASCARA \\
\hline V348 And & 57308.37877 & 0.00742 & $P$ & $\mathrm{C}$ & MASCARA \\
\hline V348 And & 57391.48638 & 0.00540 & $P$ & $\mathrm{C}$ & MASCARA \\
\hline V348 And & 56269.31277 & 0.02211 & $S$ & $\mathrm{R}$ & This study \\
\hline V348 And & 56560.38780 & 0.00064 & $P$ & $\mathrm{~V}$ & This study \\
\hline V348 And & 56629.45404 & 0.00092 & S & $\mathrm{RC}$ & This study \\
\hline V348 And & 56629.45582 & 0.00102 & S & $\mathrm{R}$ & This study \\
\hline V348 And & 56906.50226 & 0.00626 & S & BVR & This study \\
\hline V348 And & 56920.54223 & 0.00290 & $P$ & $\mathrm{R}$ & This study \\
\hline V348 And & 56934.20656 & 0.00149 & S & $\mathrm{R}$ & This study \\
\hline V348 And & 57017.30505 & 0.00101 & S & $\mathrm{R}$ & This study \\
\hline V348 And & 57031.37299 & 0.00169 & $\mathrm{P}$ & $\mathrm{C}$ & This study \\
\hline V348 And & 57266.63076 & 0.00192 & S & $\mathrm{R}$ & This study \\
\hline V348 And & 57280.69077 & 0.00202 & $\mathrm{P}$ & $\mathrm{R}$ & This study \\
\hline V348 And & 57294.34885 & 0.00453 & S & $\mathrm{R}$ & This study \\
\hline V348 And & 57308.39410 & 0.00389 & $\mathrm{P}$ & BVR & This study \\
\hline V348 And & 57626.77573 & 0.00414 & S & $\mathrm{R}$ & This study \\
\hline V348 And & 57696.24057 & 0.00211 & $\mathrm{P}$ & BVR & This study \\
\hline V348 And & 57751.65673 & 0.00088 & $P$ & $\mathrm{R}$ & This study \\
\hline V348 And & 58028.68835 & 0.00571 & $P$ & $\mathrm{R}$ & This study \\
\hline V348 And & 58042.34567 & 0.00301 & S & BVR & This study \\
\hline V348 And & 58056.38505 & 0.01229 & $\mathrm{P}$ & $\mathrm{R}$ & This study \\
\hline V348 And & 58402.47455 & 0.00145 & S & I & This study \\
\hline V348 And & 58416.53209 & 0.00800 & $\mathrm{P}$ & $\mathrm{R}$ & This study \\
\hline V572 Per & 48046.84267 & 0.00120 & $\bar{P}$ & $\mathrm{Hp}$ & Hipparcos \\
\hline V572 Per & 48585.49511 & 0.00299 & $P$ & $\mathrm{Hp}$ & Hipparcos \\
\hline V572 Per & 48586.07734 & 0.00085 & S & Hp & Hipparcos \\
\hline V572 Per & 53793.64709 & 0.00427 & $\mathrm{P}$ & $\mathrm{V}$ & OMC/INTEGRAL \\
\hline V572 Per & 53394.52152 & 0.01259 & $P$ & $\mathrm{~V}$ & OMC/INTEGRAL \\
\hline V572 Per & 57263.34037 & 0.00369 & $P$ & $\mathrm{~V}$ & OMC/INTEGRAL \\
\hline V572 Per & 57245.76753 & 0.00217 & S & $\mathrm{C}$ & MASCARA \\
\hline V572 Per & 57282.75239 & 0.00084 & $P$ & $\mathrm{C}$ & MASCARA \\
\hline V572 Per & 57317.93136 & 0.00082 & $P$ & $\mathrm{C}$ & MASCARA \\
\hline V572 Per & 57318.55851 & 0.00191 & S & $\mathrm{C}$ & MASCARA \\
\hline V572 Per & 57370.10208 & 0.00153 & $P$ & $\mathrm{C}$ & MASCARA \\
\hline V572 Per & 57438.66681 & 0.00216 & S & $\mathrm{C}$ & MASCARA \\
\hline V572 Per & 55968.29287 & 0.00112 & S & $\mathrm{C}$ & This study \\
\hline V572 Per & 55988.29012 & 0.00107 & $P$ & BVRI & This study \\
\hline V572 Per & 56008.32734 & 0.00053 & S & $\mathrm{R}$ & This study \\
\hline V572 Per & 56154.49526 & 0.00127 & $P$ & BVRI & This study \\
\hline V572 Per & 56157.54991 & 0.00111 & S & BVRI & This study \\
\hline V572 Per & 56205.44812 & 0.00026 & $P$ & $\mathrm{R}$ & This study \\
\hline V572 Per & 56225.48699 & 0.00027 & S & $\mathrm{C}$ & This study \\
\hline V572 Per & 56290.37051 & 0.00089 & $P$ & BVRI & This study \\
\hline V572 Per & 56290.37017 & 0.00028 & $P$ & I & This study \\
\hline V572 Per & 56510.58550 & 0.00066 & S & $\mathrm{V}$ & This study \\
\hline V572 Per & 56541.49831 & 0.00017 & $P$ & V & This study \\
\hline V572 Per & 56566.38983 & 0.00201 & S & BVRI & This study \\
\hline V572 Per & 56623.41085 & 0.00027 & S & $\mathrm{C}$ & This study \\
\hline V572 Per & 56643.40532 & 0.00017 & $P$ & $\mathrm{C}$ & This study \\
\hline V572 Per & 56711.34419 & 0.00016 & $P$ & $\mathrm{C}$ & This study \\
\hline V572 Per & 56891.52002 & 0.00029 & S & $\mathrm{C}$ & This study \\
\hline V572 Per & 56922.43527 & 0.00040 & $P$ & $\mathrm{C}$ & This study \\
\hline V572 Per & 56964.31399 & 0.00026 & S & I & This study \\
\hline V572 Per & 57261.53953 & 0.00406 & S & $\mathrm{C}$ & This study \\
\hline V572 Per & 57275.47175 & 0.00026 & $P$ & $\mathrm{~V}$ & This study \\
\hline V572 Per & 57340.39911 & 0.00267 & $S$ & $\mathrm{C}$ & This study \\
\hline V572 Per & 57366.46036 & 0.00025 & $P$ & $\mathrm{C}$ & This study \\
\hline V572 Per & 57594.53794 & 0.00021 & $P$ & I & This study \\
\hline V572 Per & 57614.57759 & 0.00038 & S & $\mathrm{V}$ & This study \\
\hline V572 Per & 57964.55903 & 0.00059 & $P$ & $\mathrm{C}$ & This study \\
\hline V572 Per & 58080.43684 & 0.00019 & S & $\mathrm{R}$ & This study \\
\hline V572 Per & 58376.45323 & 0.00035 & S & V & This study \\
\hline V572 Per & 58498.35689 & 0.00054 & $\mathrm{P}$ & $\mathrm{V}$ & This study \\
\hline
\end{tabular}


TABLE 4

RADIAL VELOCITIES OF THE SYSTEMS USED FOR OUR ANALYSIS.

\begin{tabular}{|c|c|c|c|c|c|}
\hline $\begin{array}{l}\text { Star } \\
\text { name }\end{array}$ & $\begin{array}{l}\text { HJD - } 2400000 \\
\text { [d] }\end{array}$ & $\begin{array}{r}\mathrm{RV}_{1} \\
{[\mathrm{~km} / \mathrm{s}]}\end{array}$ & $\begin{array}{c}\text { Error } \\
{[\mathrm{km} / \mathrm{s}]}\end{array}$ & $\begin{array}{r}\mathrm{RV}_{2} \\
{[\mathrm{~km} / \mathrm{s}]}\end{array}$ & $\begin{array}{r}\text { Error } \\
{[\mathrm{km} / \mathrm{s}]}\end{array}$ \\
\hline V348 And & 56041.5766 & -69.91 & 1.65 & 50.77 & 0.39 \\
\hline V348 And & 56357.2585 & 37.68 & 2.01 & -59.13 & 0.48 \\
\hline V348 And & 56400.6093 & -76.20 & 1.66 & 53.01 & 7.92 \\
\hline V348 And & 56534.5871 & -46.54 & 0.87 & 21.13 & 1.16 \\
\hline V348 And & 56563.6216 & -61.96 & 2.66 & 37.55 & 2.48 \\
\hline V348 And & 56572.5450 & -23.97 & 0.98 & 9.45 & 1.28 \\
\hline V348 And & 56590.2923 & -48.02 & 1.12 & 22.40 & 4.06 \\
\hline V348 And & 56590.5739 & -50.89 & 1.48 & 31.41 & 3.09 \\
\hline V348 And & 56592.3997 & -72.36 & 1.36 & 52.12 & 1.45 \\
\hline V348 And & 56596.6362 & -63.08 & 2.15 & 40.61 & 1.12 \\
\hline V348 And & 56665.1968 & 46.17 & 2.10 & -76.87 & 1.93 \\
\hline V348 And & 56665.4144 & 46.11 & 2.66 & -74.71 & 2.50 \\
\hline V348 And & 56862.4588 & 29.47 & 0.38 & -55.96 & 2.47 \\
\hline V348 And & 56924.4092 & -67.61 & 2.05 & 46.82 & 4.51 \\
\hline V348 And & 57084.2744 & 23.89 & 2.60 & -50.82 & 3.06 \\
\hline V348 And & 57248.4857 & 45.88 & 7.34 & -68.68 & 5.07 \\
\hline V348 And & 57260.5891 & -69.17 & 2.55 & 48.80 & 0.80 \\
\hline V348 And & 57275.5356 & 46.09 & 4.10 & -74.11 & 2.39 \\
\hline V348 And & 57323.5005 & 1.75 & 6.28 & -24.11 & 6.24 \\
\hline V348 And & 57328.5228 & 45.15 & 1.43 & -70.60 & 3.27 \\
\hline V348 And & 57868.5572 & -76.57 & 0.80 & 54.89 & 1.93 \\
\hline V348 And & 57876.5621 & -7.49 & 2.37 & - & \\
\hline V348 And & 57954.5020 & -61.05 & 2.28 & 38.25 & 2.76 \\
\hline V572 Per & 56571.4889 & 122.65 & 3.51 & -163.36 & 4.32 \\
\hline V572 Per & 56572.5780 & 77.87 & 3.10 & -104.99 & 2.56 \\
\hline V572 Per & 56590.3078 & -118.92 & 2.72 & 152.61 & 6.22 \\
\hline V572 Per & 56590.4916 & -90.88 & 3.93 & 117.60 & 5.50 \\
\hline V572 Per & 56592.3713 & 56.92 & 3.98 & -73.27 & 8.75 \\
\hline V572 Per & 56862.5158 & 72.87 & 2.78 & -88.93 & 2.63 \\
\hline V572 Per & 56862.5837 & 108.78 & 1.93 & -127.26 & 3.57 \\
\hline V572 Per & 56924.3582 & 59.83 & 6.17 & -71.36 & 4.16 \\
\hline V572 Per & 57079.3053 & -120.45 & 0.99 & 148.18 & 4.86 \\
\hline V572 Per & 57084.3023 & -65.68 & 1.30 & 81.68 & 5.05 \\
\hline V572 Per & 57084.3527 & -41.35 & 2.59 & 39.24 & 3.78 \\
\hline V572 Per & 57126.3372 & -56.04 & 4.50 & 64.81 & 6.40 \\
\hline V572 Per & 57260.6045 & 130.68 & 4.84 & -165.72 & 5.07 \\
\hline V572 Per & 57294.4578 & 91.56 & 1.81 & -119.09 & 2.75 \\
\hline V572 Per & 57323.4676 & 36.29 & 6.17 & -39.45 & 2.40 \\
\hline V572 Per & 57328.5854 & 128.18 & 4.15 & -165.25 & 4.62 \\
\hline V572 Per & 57330.3089 & -117.55 & 3.29 & 145.97 & 6.57 \\
\hline V572 Per & 57332.4880 & 15.51 & 2.24 & -14.78 & 1.54 \\
\hline V572 Per & 57410.4423 & -124.74 & 2.91 & 155.86 & 3.00 \\
\hline V572 Per & 57443.3294 & -99.99 & 4.43 & 127.98 & 4.09 \\
\hline V572 Per & 57876.3165 & -127.74 & 3.29 & 159.44 & 4.39 \\
\hline
\end{tabular}

